\title{
Effects of Attentional Focus during Short-Term Drop-Jump Training on Strength, Jump and Sprint Performances in Hurling Players
}

\author{
Paul J Byrne ${ }^{1,2 *}$, Jeremy A Moody², Stephen-Mark Cooper ${ }^{2}$, Michael Lawlor ${ }^{1}$ and Sharon Kinsella ${ }^{1}$ \\ ${ }^{1}$ Department of Science and Health, Institute of Technology Carlow, Ireland \\ ${ }^{2}$ Cardiff School of Sport and Health Sciences (Sport), Cardiff Metropolitan University, Cardiff, UK
}

Submission: June 05, 2018; Published: June 21, 2018

*Corresponding author: Paul J Byrne, Department of Science and Health, Institute of Technology Carlow, Kilkenny Road, Carlow, Ireland, Tel: +353599175585; Email: paul.byrne@itcarlow.ie

Abstract

The purpose of this study was to investigate the effects of attentional focus during a bounce drop-jump (BDJ) training program on the reactive strength index (RSI), maximum strength, counter movement jump (CMJ) and sprint performances in male hurling players. Twenty players were assigned to either a NONCOACH (no coaching instruction) or a $\mathrm{COACH}$ (neutral, internal and external focus coaching instructions) group and were tested at baseline, pre- and post- the 6-week BDJ program. Significant differences were found pre-to post-program in both groups for the following measures: BDJ RSI (NONCOACH: $21.1 \%$ ( $p<0.001$ ), $\mathrm{ES}=0.81$; $\mathrm{COACH}: 22.2 \%(\mathrm{p}=0.010)$, ES $=0.76)$ ) BDJ height (NONCOACH: 19.8\%(p =0.010), ES =0.91; COACH: $21.1 \%(\mathrm{p}=0.010)$, $\mathrm{ES}=0.81)$; absolute $3 \mathrm{RM}$ back squat (NONCOACH: $6.6 \%(\mathrm{p}=0.020), \mathrm{ES}=0.36$; COACH: $12.3 \%(p=0.030)$, ES $=0.79)$ and relative $3 \mathrm{RM}$ back squat (NONCOACH: $6.7 \%(\mathrm{p}=0.010)$, ES $=0.53$; COACH: $12.6 \%(\mathrm{p}=0.040)$, ES $=0.79)$.The $\mathrm{COACH}$ group increased eccentric rate of force development (ECC-RFD) from pre- to post-program (absolute ECC-RFD: $44.6 \%$ ( $p=0.050), \mathrm{ES}=$ 0.60; relative ECC-RFD: $44.7 \%$ ( $p=0.040)$, ES $=0.58$ ), from baseline to post-program for CMJ height $(9.5 \%(p=0.020)$, ES $=0.45)$ and peak force (absolute: $11.2 \%(\mathrm{p}=0.040)$, ES $=0.70$; relative: $10.1 \%(\mathrm{p}=0.040)$, ES $=0.67)$. Including coaching instructions during BDJ training is effective in enhancing strength and power capacities in hurling players and is transferrable to jumping.

Keywords: Drop-jump; Potentiation; Attentional focus; Stretch-shortening cycle; Hurling

Abbreviations: DJ: Drop-Jumping; GRF: Ground Reaction Forces; SSC: Stretch-Shortening Cycle; BDJ: Bounce Drop Jump; RSI: Reactive Strength Index; CMJ: Counter Movement Jump; 3RM: Three Repetition Maximum

\section{Introduction}

Drop-jumping (DJ) was developed as an exercise to strengthen the working muscles and improve an athlete's reactive ability where the amortization phase (ground contact time) is minimized [1]. Furthermore, DJs demand the dynamic strength to withstand landing ground reaction forces (GRF) and the reactive ability of the neuromuscular system to tolerate a relatively high stretch load and change from a rapid eccentric muscle action to a rapid concentric muscle action, i.e. a fast stretch-shortening cycle (SSC) [2]. To perform a DJ, athletes are required to drop from a predetermined height and on landing perform a subsequent vertical or horizontal jump. A DJ technique, related to the fast SSC has been proposed and termed a bounce DJ (BDJ) which employs small angular displacements of the hip, knee and ankle joints resulting in reduced ground contact times and high GRFs [3]. As the BDJ is associated with a fast SSC, ground contact time between the eccentric and concentric phases of the SSC is $\leq 0.250$ $s$ [4]. A key benefit of BDJs using the fast SSC is enhanced power production. There is research available into the ability of BDJs to enhance reactive strength and power production [5-9].

DJ training has been shown to be effective in improving the strength, jump and sprint abilities of athletes using pre-set drop heights that were not individualized [6-8]. In these studies, participants were instructed to perform the DJs to minimize contact time and maximize height, but no detail was provided about when and how these instructions were given. To date, only two studies have reported applying the RSI as a means of determining the drop height for DJ training [5,9]. Young et al. [9] reported a non-significant increase in vertical jump height and a significant increase in reactive strength for the group that performed DJs using a bounce technique where ground contact time $<0.250 \mathrm{~s}$ and height maximized. Byrne et al. [5] also reported a significant improvement in reactive strength but, in contrast to the results of Young et al. [9], this resulted in a significant improvement in CMJ height. However, no study to date, has examined the effect of 
coaching instructions during a short-term DJ program to enhance the bounce technique.

From a coaching perspective, coaching instructions and cues facilitate attentional focus; defined as the conscious ability of an individual to focus their attention through particular thoughts in an attempt to perform a task [10]. An individual can focus their attention internally on the movements of their body; externally focusing the effect movements have on the environment or neutrally where there is no attempt at conscious focus but instead, non awareness is encouraged [11-13]. A study by Young et al. [14] used three sets of instructions (maximize jump height, minimize contact time, or, minimize contact time and maximize jump height) and reported significant differences between jump heights, contact time and RSI between three sets. In the study by Khuu et al. [15], the use of three coaching instructions (minimize ground contact time, maximize jump height, or, synchronously extend the lower extremity joints) was also examined. Results showed that when instructed to 'minimize ground contact time', participants landed more stiffly in comparison to when the other instructions were given. This led to attenuated jump height but augmented performance variables (RSI, rate of force development, peak force and peak power). When considering the acute effect of coaching instructions on vertical jumping and standing long jump, previous research has found that external focus leads to the greatest improvement in performance of these jumps in comparison to internal focus coaching instructions [16-18]. However, the study by Wu et al. [17] found that internal focus instructions led to a greater enhancement of peak force in comparison to external focus instructions, but this did not lead to enhanced distance achieved. In a longitudinal study, Makaruk et al. [19] examined how attentional focus over a 9-week plyometric program would impact upon jumping performance. Results showed that DJ height increased significantly for the three experimental groups (control, external focus and internal focus), however, the control group achieved the greatest improvements pre- to post- the 9-week program. When considering DJ contact time, only the control group displayed a significant improvement from pre-to post-the 9-week training program. It is important to note that the DJ used in this study was a countermovement DJ due to the contact times being $>0.250 \mathrm{~s}$. This provides an indication of the functioning of the slow SSC [4].

Furthermore, the plyometric program was a mixture of plyometric exercises that were performed at various phases of the program [19].To the best of our knowledge, no study has examined the effects of attentional focus strategies during a BDJ training program, how this influences the BDJ as well as the transfer effect to strength, CMJ and sprinting performances. The present study investigated the use of coaching instructions recommended by Flanagan and Comyns [20] that are a combination (neutral, internal and external) of attentional focus instructions. These instructions were investigated as they are recommended to assist athletes in their enhancement of the BDJ technique. Therefore, the purpose of the present study was to investigate the effect of a combination of attentional focus coaching instructions during short-term BDJ training on BDJ performance, CMJ performance, 3RM back squat strength and sprint acceleration over $5 \mathrm{~m}, 10 \mathrm{~m}$ and $20 \mathrm{~m}$ in hurling players.

\section{Methods}

\section{Experimental Design}

The current study was conducted to determine the effect of recommended coaching instructions for fast SSC exercises, BDJs during a short-term training program. Hurling players were examined because the ability to jump and sprint is essential for successful performance in this field sport. A counter balanced group design was employed using RSI with repeated measures and participants acting as their own control. Participants were allocated to one of two groups: A NONCOACH (no coaching instruction given) and a $\mathrm{COACH}$ (received combination of neutral, internal and external focus coaching instructions) group. Groups were counterbalanced based upon participants' best RSI scores from the drop-jump test for the 6-week BDJ program. Participants were tested on three occasions: a baseline test (control), 5-weeks later as a pre-test and post-tested after the 6-week BDJ program. Measures for CMJ, RSI, sprint performances over $5 \mathrm{~m}, 10 \mathrm{~m}$ and $20 \mathrm{~m}$, and 3RM back squat strength were collected during each of these testing sessions. The training program commenced after the pre-test. Pre- and post-testing were completed within seven days of the commencement of, and completion of, the training program. Participants continued their usual hurling training during the 5-week lead-in time to the pre-test.

\section{Participants}

Twenty male college-level hurling players (mean \pm SD: age $=21.2 \pm 2.1$ years; mass $=77.8 \pm 4.9 \mathrm{~kg}$; height $=179.7 \pm 4.0 \mathrm{~cm}$ ) volunteered to participate in the study of the 20 players, 13 completed a 6-week BDJ training program. Participant withdrawal was due to injury incurred during competitive hurling matches or other commitments that prevented players from completing the full training program. Players had on average 13 years' game experience, four years of weight training experience and one year of plyometric training experience. However, participants had no previous experience of BDJ training.The training program was completed during the players' pre-season whilst they were involved in five hours of hurling training per week alongside the DJ training program. No orthopedic or musculoskeletal injuries to the lower extremities in the previous six months were reported during medical screening. Written informed consent was provided by all participants before testing commenced. Ethical approval was obtained from the principal investigator's institutional ethics committee.

\section{Procedures}

Participants were familiarized with both the testing and training protocols during a single session which occurred a week before baseline testing. To account for diurnal variations, participants were tested and completed the BDJ program, indoors, 
at the same time of day. The same footwear was required for all tests and training, and a dynamic warm-up was performed before participating in all testing and training sessions [21].

\section{Coaching Instructions and the Bounce Drop Jump (BDJ) Training Program}

The NONCOACH group did not receive coaching instructions for the duration of the study. The COACH group received verbal coaching instructions pertaining to the performance of the jumps during each training session. These instructions were:

a) 'minimize ground contact time and maximize jump height';

b) 'visualize the leg as a stiff spring that rebounds off the surface on landing';

c) 'Pre-tense the leg muscles before landing' [20].

These instructions were provided as feedback based upon the judgment of the principal investigator and depending upon the jumps performed by the participants. Both groups were supervised throughout the training program by the principal investigator and a sport science intern. The training program employed the BDJ technique [3] and was comprised of two sessions a week, at least 48 hours apart [22], over 6-weeks [23], where participants performed 6 sets of 10 repetitions per session [8]. Recovery times of 15 seconds were allowed between BD] repetitions and 2 minutes between sets [24].

\section{Reactive Strength Index(RSI) Testingand Determination of Drop Height}

Participants performed a BDJ test to determine their maximum RSI, which was then used to identify individualized drop heights for the BDJ training. Two BDJs from five different drop heights were performed onto a portable force plate $(0.20 \mathrm{~m}, 0.30 \mathrm{~m}, 0.40 \mathrm{~m}$, $0.50 \mathrm{~m}$ and $0.60 \mathrm{~m}$ ). In order for the stretch load (intensity) to be progressively increased, an incremental protocol was employed because familiarization of the BDJs, and 2minutes' rest between drop heights to minimize fatigue, was built into the protocol as the potential for order-effects was considered insignificant [9]. The highest RSI from two BDJs for each drop height was used for analysis. Individualized drop height was determined by employing the RSI method, which identifies the drop height that produces the maximum RSI [5]. Objectively, the ground contact time for each BDJ jump had to be $\leq 0.250 \mathrm{~s}$ [4].

\section{Countermovement Jump (CMJ) Testing}

CMJ testing required participants to perform three CMJs by squatting to a self-selected depth with hands placed on the hips and then jumping upward for maximum height, taking-off and landing on a portable force plate (Type 92886AA, Kistler Instruments Ltd, Hook, United Kingdom). Testing consisted of three trials, based upon height jumped; the best trial was used for analysis.

\section{Data Analysis for CMJ, Reactive Strength Testing and Drop Height Determination}

A portable multi-component force plate with a built-in charge amplifier (Type 92886AA, Kistler Instruments Ltd, Hook, United Kingdom) was used to collect force-time measures at a sampling frequency of $1000 \mathrm{~Hz}$. Using a bespoke protocol, data was saved and analyzed using BTS-SMART software (BTS Spa, Milan, Italy). CMJ variables included jump height, peak velocity, peak force, peak power and average eccentric rate of force development (ECCRFD) calculated as absolute and relative values (to body mass (kg)) except for jump height and peak velocity. Peak force was the highest GRF in the vertical component during the concentric phase of the jump. Peak power was calculated from the product of peak force and peak velocity of the center of mass from the CMJ. To calculate peak velocity, center of mass velocity was derived from the numerical integration of vertical acceleration. Vertical acceleration was calculated by dividing the vertical ground reaction force by the subject's body mass. ECC-RFD was calculated during the eccentric phase of the CMJ from the force-time curve when force exceeds body weight $(\mathrm{N})$ and ends when velocity is equal to zero (bottom of descent prior to moving in an upward direction towards take-off) [25]. Jump height for the CMJ and BDJ was calculated from flight time using the following equation: [26] $\mathrm{H}=\left(\mathrm{g} \cdot \mathrm{t}^{-2}\right) / 8 \quad$ where: $\mathrm{H}=$ jump height $(\mathrm{m}) ; \mathrm{g}$ =acceleration due to gravity $\left(9.81 \mathrm{~m} \mathrm{~s}^{-2}\right)$; $\mathrm{t}=$ flight time $(\mathrm{s})$. Ground contact time during the amortization phase (the time-frame that the subject is in contact with the ground before the subsequent jump) was calculated as the time between initial foot contact and take-off [27]. The RSI was calculated based on the equation: RSI = jump height $(\mathrm{m}) /$ contact time $(\mathrm{s})$.

\section{Sprint Performance Testing}

Starting in a static upright position $0.5 \mathrm{~m}$ behind the first timing photocell (Microgate, Bolzano, Italy), participants performed three maximal sprint trials over $20 \mathrm{~m}$ spaced three minutes apart. Photocells were also placed at $5 \mathrm{~m}$ and $10 \mathrm{~m}$ in order to record split-times, and at $20 \mathrm{~m}$ to record maximal sprint times. Times from the best trial were used for analysis.

\section{Three Repetition Maximum (3RM) Back Squat Strength Testing}

Strength testing was conducted after a five-minute recovery period following the end of the $20 \mathrm{~m}$ sprint testing protocol. A modified form of a three repetition maximum (3RM) protocol was used [28], commencing with a warm-up comprising of two sets of eight repetitions at $50 \%$ of the participants' predicted 1RM. This was followed by four repetitions at $70 \%$ of predicted $1 \mathrm{RM}$. After completing the four repetitions, participants attempted performing three repetitions at a 3RM load. A two-minute and a five-minute recovery period were allowed between warm-up sets and 3RM attempts respectively. Participants were required to squat down until their thighs were parallel with the ground; the position being set by a bench placed behind the participant. The 
3RM trials continued until the participant was unable to complete the lift through the designated range of movement. Relative strength was calculated as: relative strength $=3 \mathrm{RM}(\mathrm{kg}) /$ body mass $(\mathrm{kg})$.

\section{Statistical Analyses}

Data are summarised as means \pm SDs, provided for all measures. Seven participants that withdrew from the study were excluded so that comparisons would not be skewed. A three (time) by two (groups: NONCOACH vs $\mathrm{COACH}$ ) within-between analysis of variance (ANOVA) was used to identify significant differences between means for all the dependent variables. For RSI, CMJ peak force, $5 \mathrm{~m}, 10 \mathrm{~m}$ and $20 \mathrm{~m}$ sprint times, a Greenhouse-Geisser adjustment was applied because Mauchleys test for sphericity was violated. Effect sizes (partial eta) and power are also provided for each of the comparisons. Repeated measures ANOVAs were performed for both groups on means for RSI, BDJ height and 3RM back squat strength (absolute and relative) followed by paired $t$-tests (with Dunn-Sidak adjustment) as post-hoc pair wise comparisons. For pair wise comparisons for CMJ measures, paired t-tests were conducted to identify significant differences pre- to post-training on absolute and relative ECC-RFD. Wilcoxon's signed-rank tests were applied for absolute and relative peak force, and CMJ height to identify significant differences from baseline to post-training. Effect size was calculated using Cohen's $d$ where the mean of the differences between the pre-test and the post-test scores were divided by the respective pooled SD. Effect sizes were interpreted as $<0.2=$ trivial, 0.2-0.5 =small, 0.5 $0.8=$ moderate, and $0.8 \geq=$ large [29]. The sprint and 3RM back squat strength measures were found to be reliable using an intra class correlation coefficient ( $5 \mathrm{~m}$ : ICC $=0.90 ; 10 \mathrm{~m}$ : $=0.95 ; 20 \mathrm{~m}$ : $=0.96$; absolute and relative $3 \mathrm{RM}:=0.99$ ). The reliability of the $\mathrm{CMJ}$ and drop jump measures have been previously established [30]. Statistical significance was set atp $\leq 0.05$ throughout and all statistical analyses were conducted using the Statistical Package for the Social Sciences version 23 (SPSS Inc., Chicago, Illinois).

\section{Results}

Table 1: BDJ test measures (mean \pm SD) and effect sizes (ES) for baseline, pre- and post- the 6-week BDJ training programme for the $\mathrm{NONCOACH}(n=7)$ and $\mathrm{COACH}(\mathrm{n}=6)$ groups.

\begin{tabular}{|c|c|c|c|c|}
\hline Variable & Baseline & Pre-test & Post-test & ES \\
\hline \multicolumn{5}{|c|}{ RSI (ms $^{-1}$ ) } \\
\hline NONCOACH & $1.43 \pm 0.50$ & $1.26 \pm 0.31$ & $1.50 \pm 0.29^{*}$ & 0.76 \\
\hline COACH & $1.19 \pm 0.55$ & $1.11 \pm 0.46$ & $1.33 \pm 0.50^{*}$ & 0.47 \\
\hline \multicolumn{5}{|c|}{ Jump height (cm) } \\
\hline NONCOACH & $25.07 \pm 5.98$ & $23.01 \pm 4.42$ & $27.31 \pm 4.19^{*}$ & 0.91 \\
\hline COACH & $22.51 \pm 6.92$ & $22.06 \pm 5.15$ & $26.36 \pm 4.87^{*}$ & 0.81 \\
\hline \multicolumn{5}{|c|}{ Contact time (s) } \\
\hline NONCOACH & $0.181 \pm 0.025$ & $0.186 \pm 0.028$ & $0.182 \pm 0.009$ & -0.17 \\
\hline COACH & $0.195 \pm 0.034$ & $0.211 \pm 0.031$ & $0.207 \pm 0.035$ & -0.11 \\
\hline
\end{tabular}

*denotes a significant difference between pre-test and post-test means $(p \leq 0.05)$.
Before the training program commenced, groups were compared to ensure they were counterbalanced based upon their RSI. Baseline and pre-training RSI scores were shown to be not normally distributed. Mann Whitney $\mathrm{U}$ tests between groups showed non-significant differences for the RSI baseline ( $p>0.05$ ) and pre-training scores ( $p>0.05)$. There were non-significant outcomes for the groups' main effect and for the time $\mathrm{x}$ groups' interaction effect for all drop-jump tests measures (RSI, BDJ height and ground contact time), all CMJ measures, 3RM back squat strength measures (absolute and relative) and all the sprint performance measures ( $\mathrm{p}>0.05)$.

Table 2: CMJ test measures (mean \pm SD) and effect sizes (ES) for baseline, pre- and post- the 6-week BDJ training programme for the $\mathrm{NONCOACH}(n=7)$ and $\mathrm{COACH}(n=6)$ groups.

\begin{tabular}{|c|c|c|c|c|}
\hline Variable & Baseline & Pre-test & Post-test & ES \\
\hline \multicolumn{5}{|c|}{ Height (cm) } \\
\hline NONCOACH & $38.67 \pm 3.57$ & $39.1 \pm 5.53$ & $40.1 \pm 4.09$ & 0.21 \\
\hline $\mathrm{COACH}$ & $34.22 \pm 6.83$ & $34.97 \pm 6.75$ & $37.51 \pm 7.84^{*}$ & 0.35 \\
\hline \multicolumn{5}{|c|}{ Velocity $\left(\mathrm{ms}^{-1}\right)$} \\
\hline NONCOACH & $2.94 \pm 0.41$ & $2.95 \pm 0.40$ & $2.92 \pm 0.32$ & 0.35 \\
\hline $\mathrm{COACH}$ & $2.78 \pm 0.22$ & $3.01 \pm 0.63$ & $3.01 \pm 0.58$ & 0.58 \\
\hline \multicolumn{5}{|c|}{ Peak Force (N) } \\
\hline NONCOACH & $1777 \pm 396$ & $1815 \pm 369$ & $1839 \pm 410$ & 0.06 \\
\hline $\mathrm{COACH}$ & $1726 \pm 200$ & $1715 \pm 203$ & $1916 \pm 331 *$ & 0.76 \\
\hline \multicolumn{5}{|c|}{ Peak force $\left(\mathrm{N} \mathrm{kg}^{-1}\right)$} \\
\hline NONCOACH & $2.35 \pm 0.19$ & $2.4 \pm 0.14$ & $2.43 \pm 0.18$ & 0.18 \\
\hline $\mathrm{COACH}$ & $2.37 \pm 0.27$ & $2.29 \pm 0.23$ & $2.54 \pm 0.40^{*}$ & 0.81 \\
\hline \multicolumn{5}{|c|}{ Peak power $(W)$} \\
\hline NONCOACH & $5213 \pm 1194$ & $5398 \pm 1465$ & $5433 \pm 1662$ & 0.02 \\
\hline $\mathrm{COACH}$ & $4784 \pm 602$ & $5038 \pm 1426$ & $5788 \pm 1740$ & 0.48 \\
\hline \multicolumn{5}{|c|}{ Peak power $\left(\mathrm{W} \mathrm{kg}^{-1}\right)$} \\
\hline $\begin{array}{c}\text { Non- } \\
\text { Coached }\end{array}$ & $67.85 \pm 9.68$ & $69.61 \pm 11.05$ & $69.58 \pm 12.23$ & -0.002 \\
\hline Coached & $62.54 \pm 8.68$ & $65.97 \pm 21.61$ & $75.85 \pm 25.50$ & 0.43 \\
\hline \multicolumn{5}{|c|}{ ECC-RFD $\left(\mathrm{N} \mathrm{s}^{-1}\right)$} \\
\hline NONCOACH & $5631 \pm 3121$ & $5786 \pm 2990$ & $6562 \pm 2438$ & 0.24 \\
\hline $\mathrm{COACH}$ & $4118 \pm 1537$ & $4094 \pm 2142$ & $5429 \pm 2739 * *$ & 0.60 \\
\hline \multicolumn{5}{|c|}{ ECC-RFD $\left(\mathrm{N} \mathrm{kg}^{-1}\right)$} \\
\hline NONCOACH & $6.30 \pm 3.51$ & $6.53 \pm 3.30$ & $7.60 \pm 3.06$ & 0.34 \\
\hline $\mathrm{COACH}$ & $5.33 \pm 2.01$ & $5.28 \pm 2.93$ & $7.17 \pm 3.60^{* *}$ & 0.58 \\
\hline
\end{tabular}

*denotes a significant difference between baseline and post-test means $(p<0.05)$.

${ }^{* *}$ denotes a significant difference between pre- and post-test means $(p<0.05)$. 
Drop Jump Test (RSI, Jump Height and Ground Contact Time)

Table 1 and Figure 1 summarize the results for BDJ RSI, jump height and ground contact time at baseline, pre- and postthe 6-week BDJ training program for both the NONCOACH and the COACH groups. A significant effect for time was shown for
RSI $\left(F_{1.32,14.61}=11.08(\mathrm{p}=0.003)\right.$; partial eta $=0.50$; power $\left.=0.92\right)$ and jump height $(F=10.83(\mathrm{p}=0.001)$; partial eta $=0.49$; power $=0.97)$. Pair-wise comparisons showed a significant increase for NONCOACH RSI $(21.1 \%(\mathrm{p}<0.001)$; ES $=0.91)$, COACH RSI $(22.2$ $\%(p=0.01)$; ES = 0.76), NONCOACH BDJ height (19.8 \% ( $p=0.01)$; $\mathrm{ES}=0.91)$ and COACH BDJ height $(21.1 \%(\mathrm{p}=0.01) ; \mathrm{ES}=0.81)$ pre- to post-the BDJ program.

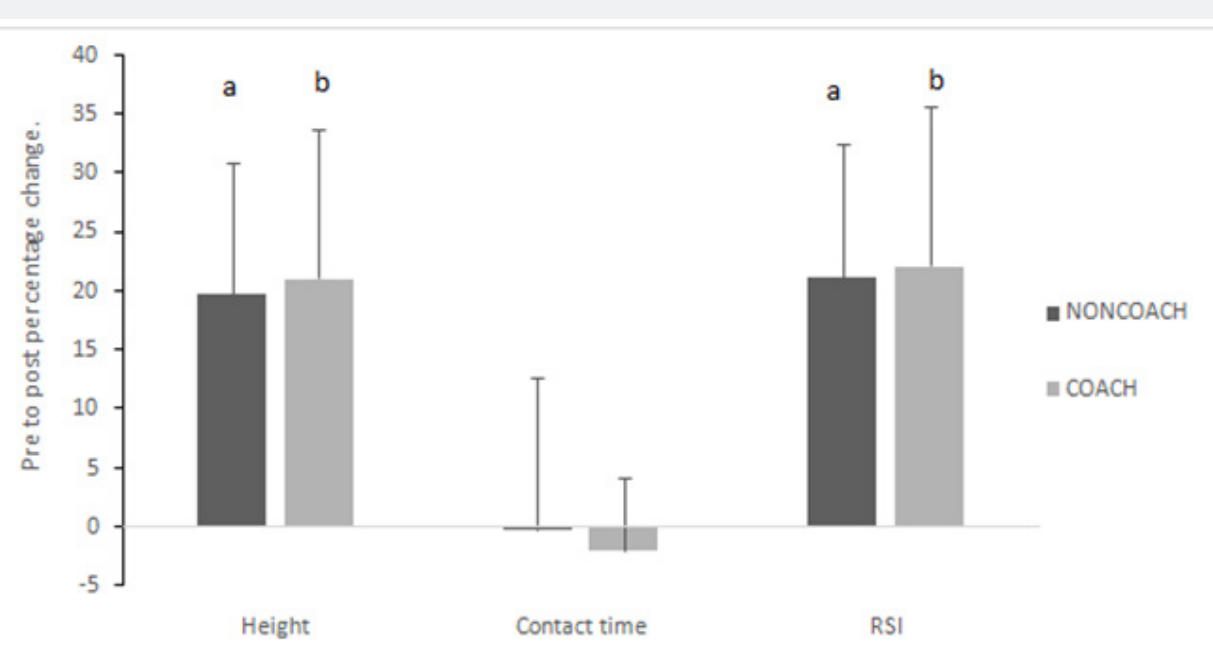

Figure 1: Pre- to post-training percentage changes (mean $\pm \mathrm{SD}$ ) for BDJ height, ground contact time and RSI for the NONCOACH and $\mathrm{COACH}$ groups.a) denotes a significant difference $(p<0.01)$. b) denotes a significant difference $(p<0.05)$.

Table 3: 3RM strength test measures (mean $\pm \mathrm{SD}$ ) and effect sizes $(\mathrm{ES})$ for baseline, pre- and post- the 6-week BDJ training programme for the $\mathrm{NONCOACH}(\mathrm{n}=7)$ and $\mathrm{COACH}(\mathrm{n}=6)$ groups.

\begin{tabular}{|c|c|c|c|c|}
\hline Variable & Baseline & Pre-test & Post-test & ES \\
\hline \multicolumn{5}{|c|}{ 3RM (kg) } \\
\hline NONCOACH & $110.00 \pm 17.55$ & $110.00 \pm 20.81$ & $117.50 \pm 24.53^{*}$ & 0.37 \\
\hline COACH & $106.66 \pm 17.22$ & $107.5 \pm 15.08$ & $120.00 \pm 13.78^{*} \uparrow$ & 0.79 \\
\hline \multicolumn{5}{|c|}{ 3RM (kg BM(kg) $\left.{ }^{-1}\right)$} \\
\hline NONCOACH & $1.45 \pm 0.21$ & $1.43 \pm 0.16$ & $1.53 \pm 0.17^{*}$ & 0.53 \\
\hline COACH & $1.42 \pm 0.32$ & $1.41 \pm 0.27$ & $1.57 \pm 0.23^{*}$ & 0.59 \\
\hline
\end{tabular}

*denotes a significant difference between pre-test and post-test means $(p \leq 0.05)$.

tdenotes a significant difference between baseline and post-test means $(p \leq 0.05)$.

\section{CMJ Measures}

Table 2 and Figure 2 summarize the results for baseline, pre- and post- 6-week BDJ training program scores for the $\mathrm{CMJ}$ measures for the NONCOACH and COACH groups. When considering the main effect for time, significant changes for the $\mathrm{COACH}$ group were identified for jump height $\left(F_{2,22}=4.92(\mathrm{p}\right.$ $=0.01)$; partial eta $=0.30$; power $=0.74)$, peak force $(F 1.15,12.69$ $=5.22(\mathrm{p}=0.03)$; partial eta $=0.32$; power $=0.59)$, relative peak force $\left(F_{1.10,12.18}=4.84(\mathrm{p}=0.04)\right.$; partial eta $=0.30$; power $\left.=0.54\right)$, eccentric $\operatorname{RFD}\left(F_{2,22}=5.1(p=0.01)\right.$; partial eta $=0.31$; power $\left.=0.76\right)$ and relative eccentric $\operatorname{RFD}\left(F_{2,22}=5.04(p=0.01)\right.$; partial eta $=0.31$; power $=0.76)$. There was also a significant increase in ECC-RFD (absolute: $44.6 \%(\mathrm{p}=0.05)$; ES $=0.60$; relative: $44.7 \%(\mathrm{p}=0.04)$; $\mathrm{ES}=0.58)$ from pre- to post- test the 6-week BDJ program. CMJ height $(9.5 \%(p=0.02)$; ES $=0.45)$, absolute peak force $(11.2 \%$ $(p=0.04), E S=0.70)$ and relative peak force $(10.1 \%(p=0.04), E S$ $=0.67$ ) increased significantly from baseline to post-testing. 


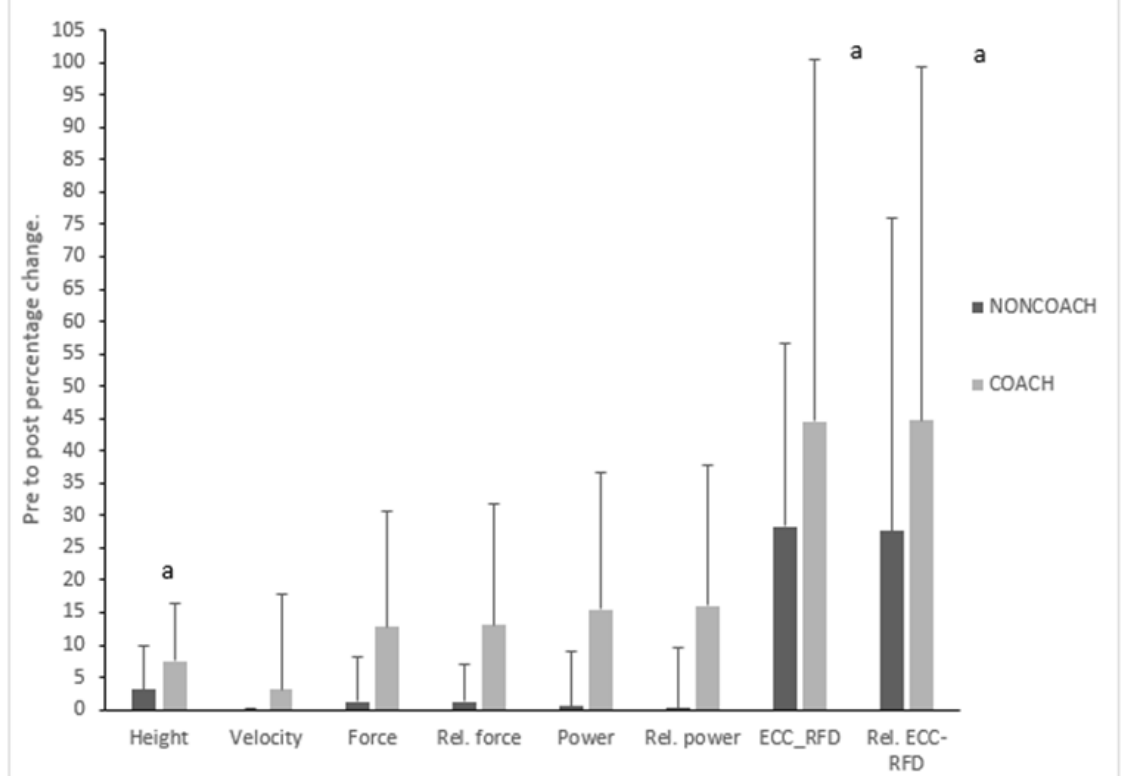

Figure 2: Pre- to post-training percentage changes (mean $\pm \mathrm{SD}$ ) for $\mathrm{CMJ}$ measures for the NONCOACH and $\mathrm{COACH}$ groups. a) denotes a significant difference $(p<0.05)$.

Table 4: Sprint test measures (mean $\pm \mathrm{SD}$ ) for baseline, pre- and post- the 6-week BDJ training programme for the NONCOACH $(n=7)$ and $\mathrm{COACH}(\mathrm{n}=6)$ groups.

\begin{tabular}{|c|c|c|c|}
\hline Variable & Baseline & Pre-test & Post-test \\
\hline \multicolumn{4}{|c|}{$5 \mathrm{~m}(\mathrm{~s})$} \\
\hline NONCOACH & $1.08 \pm 0.03$ & $1.09 \pm 0.03$ & $1.06 \pm 0.03$ \\
\hline $\mathrm{COACH}$ & $1.14 \pm 0.08$ & $1.12 \pm 0.09$ & $1.12 \pm 0.08$ \\
\hline \multicolumn{4}{|c|}{$10 \mathrm{~m}(\mathrm{~s})$} \\
\hline NONCOACH & $1.83 \pm 0.03$ & $1.83 \pm 0.02$ & $1.83 \pm 0.06$ \\
\hline $\mathrm{COACH}$ & $1.89 \pm 0.09$ & $1.90 \pm 0.10$ & $1.90 \pm 0.08$ \\
\hline \multicolumn{4}{|c|}{$20 \mathrm{~m}(\mathrm{~s})$} \\
\hline NONCOACH & $3.15 \pm 0.05$ & $3.14 \pm 0.05$ & $3.14 \pm 0.09$ \\
\hline $\mathrm{COACH}$ & $3.22 \pm 0.14$ & $3.24 \pm 0.16$ & $3.23 \pm 0.13$ \\
\hline
\end{tabular}

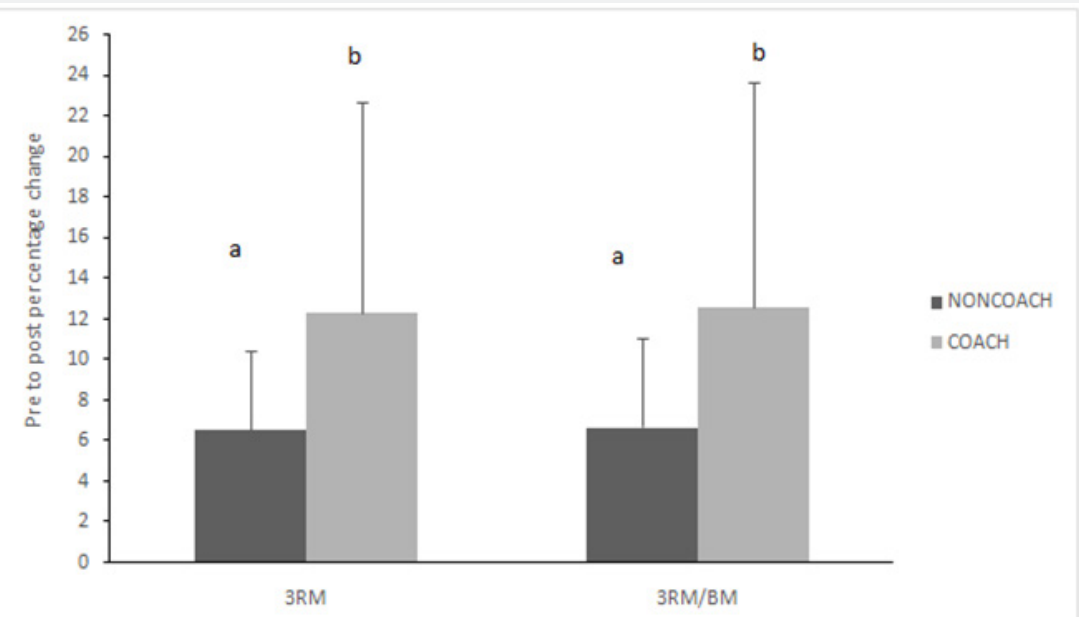

Figure 3: Pre- to post-training percentage changes (mean $\pm \mathrm{SD}$ ) for absolute and relative 3RM back squat strength for the NONCOACH and $\mathrm{COACH}$ groups. $a)$ and $b)$ denote significant differences $(p<0.05)$. 


\section{RM Back Squat Strength}

Table 3 and Figure 3 summarize the results for the absolute and relative $3 \mathrm{RM}$ back squat strength scores for the NONCOACH and $\mathrm{COACH}$ groups. A significant change for time was observed for absolute $(F 2,22=8.55(\mathrm{p}=0.002)$; partial eta squared $=0.43$; power $=0.94)$ and relative $\left(F_{2,22}=7.79\right.$ ( $\left.\mathrm{p}=0.003\right)$; partial eta $=0.41$; power $=0.91$ ) $3 \mathrm{RM}$ back squat strength. A significant increase in absolute 3RM back squat strength for both the $\mathrm{COACH}$ group (12.3\% ( $\mathrm{p}=0.03)$; ES =0.79) and for the NONCOACH group (6.6\% $(\mathrm{p}=0.02)$; ES =0.36); and relative 3RM back squat strength $(\mathrm{COACH}=12.6 \%$ ( $\mathrm{p}=0.04)$; ES $=0.58$, and NONCOACH $=6.7 \%$ (p $=0.01) ; \mathrm{ES}=0.53$ ), pre- to post- the BDJ training program.

\section{Sprint Performance}

Finally, Table 4 summarizes the results for the $5 \mathrm{~m}, 10 \mathrm{~m}$ and $20 \mathrm{~m}$ sprint times for baseline, and pre- and post- training for the NONCOACH and the COACH groups. There was no significant change for the time effect ( $p>0.05)$.

\section{Discussion}

The current study was a novel investigation into the effect of neutral, internal and external focus coaching instructions for BDJs over a 6-week training period and to examine if the addition of coaching instructions would enhance RSI, maximum strength, jumping and sprinting performance. There was no significant difference between the $\mathrm{COACH}$ and NONCOACH groups for strength, jump and sprint performance. However, the use of the combination of coaching instructions ("minimum contact time and maximum height", "pre-tense the leg muscles before landing" and "visualize the leg as a stiff spring that rebounds off the surface on landing") in the COACH group led to a significantly greater enhancement from pre- to post- the BDJ program for absolute and relative ECC-RFD. Furthermore, CMJ height and peak force (absolute and relative) were significantly enhanced from baseline to post-training. 3RM back squat strength increased significantly pre- to post- the BDJ program for both groups, however, the $\mathrm{COACH}$ group increased by double that of the NONCOACH group. BDJ height and RSI were enhanced similarly for both the NONCOACH and $\mathrm{COACH}$ groups suggesting that the coaching instructions did not cause an effect on BDJ performance. Therefore, the BDJ program employing individualized drop height based upon RSI improved the functioning of the fast SSC in both groups. The BDJ test showed RSI (NONCOACH: 21\%, COACH: 22\%) and BDJ height (NONCOACH: $20 \%, \mathrm{COACH}: 21 \%$ ) at the trained individualized drop height were significantly enhanced similarly for both groups pre- to post- the BDJ program. Furthermore, ground contact times at the trained height for both groups improved non-significantly. Consequently, BDJ height was the key measure for improving RSI in both groups. Our significant RSI improvements in both groups are in agreement with previous studies where the improvement ranged from 20 to $27 \%[5,9]$.

Despite the lack of coaching instruction effect in the $\mathrm{COACH}$ group for BDJ height, our findings are in agreement with Makaruk et al. [19] where BDJ height increased significantly. Furthermore, the COACH group improved by $22 \%$ in comparison to $13 \%$ in the Makaruk et al. [19] study. Our superior improvement in BDJ height may be a result of employing an individualized BDJ drop height. Recent work has provided evidence recommending drop-jump drop height to be individualized [31]. However, when considering attentional focus, Makakruk et al. [19] reported that the neutral, internal and external focus strategies caused an improvement in DJ height; however, the neutral group experienced the greatest improvement followed by the external focus group. It is difficult to compare our study to Makaruk et al. [19] for BDJ height as we included a control group that did not receive coaching instruction whereas the aforementioned study used neutral coaching instructions as their control. In contrast, our study employed a combination of neutral, internal and external attentional focus strategies and the coaching instruction of "minimum contact time, maximum jump height" was used the majority of the time with the instructions "pre-tense the leg muscles before landing" and "visualize the leg as a stiff spring that rebounds off the surface on landing" employed to a lesser extent than "minimum contact time, maximum jump height".

When reflecting on ground contact time, the current study found marginal improvements whereas Makaruk et al. [19] reported a significant reduction pre- to post-training for the neutral focus group and this group was significantly reduced in comparison to the external focus group. Both our results and the aforementioned study support the use of a neutral attentional focus strategy as improvements were observed in DJ height and ground contact time. However, it should be noted that Makaruk et al. [19] used the instruction, "jump as high as you can" versus our instruction "minimum contact time maximum jump height". Previous research has shown that when participants are instructed to jump for height in a DJ, the focus is on maximizing height and ground contact times are $\sim 0.400$ seconds which employs the slow SSC [4,14]. Furthermore, Makaruk et al. [19] states that a DJ with a fast SSC was employed but their reported ground contact times employed a slow SSC $(0.370$ (neutral focus group) to 0.420 (external focus group) seconds). The contrasting findings between our study and Makaruk et al. [19] are probably due to methodological differences and the participants that were studied. Makaruk et al. [19] did not limit knee flexion or ground contact time during DJ testing whereas we enforced a maximum ground contact time of $0.250 \mathrm{~s}$ during BDJ testing. Furthermore, the aforementioned study employed physical education students who may have ranged from untrained to trained whereas our hurling players had an average of 11 years playing the game and speculating may have adapted to high-velocity movements enabling them to perform the BDJs with a ground contact time of $\leq 0.250$ s.

The contrasting results between our study and Makaruk et al. [19] may have occurred because participants in the COACH group focused on enhancing jump height by applying part of the neutral 
instruction, "minimum contact time and maximum jump height" when performing the BDJ technique. To support our conclusion, Taube et al. [32] reported a non-significant improvement in BDJ height and a decrease in ground contact time for the experimental group that performed DJs from a set drop height of $0.30 \mathrm{~m}$. In this study, participants were instructed to "rebound as fast and as high as possible" but it was suggested that participants did not employ this instruction completely. Poolton et al. [33] has suggested that players, in our case hurling players, may seek the most efficient sources of information and do not follow coaching instructions provided despite frequent use during a training session. Based upon the response to coaching instructions, it may not be the instructions that are completely responsible for adaptations that occur but drop height plays a major role [32]. An alternative explanation to our results is that the order of minimum contact time and maximum jump height may have caused the BDJ height and ground contact time results. Further research should examine if an order effect is present in coaching instructions for DJ training programs.

Our BDJ height and ground contact time results appear not to be consistent with the constrained action hypothesis [34]. This hypothesis states that directing attention internally causes participants to become consciously aware of the movements of their bodies which can constrain motor behaviors. Previous research has demonstrated that inducing an internal focus of attention depresses the motor control system by reducing the efficiency of muscle fiber recruitment [35,36]. However, this hypothesis appears not to have occurred as the BDJ employs a fast SSC [20] and the success of a fast SSC is determined by the speed of its performance [37]. Nonetheless, the use of neutral, internal and external focus coaching instructions in the $\mathrm{COACH}$ group caused superior enhancement of maximum 3RM strength, CMJ peak force and ECC-RFD in contrast to the NONCOACH group. These strength and peak force results are similar to Newton et al. [38], who demonstrated that ballistic training improved CMJ average force but DJ force measures remained constant. The mechanisms behind these enhancements to BDJ height and RSI may be due to augmented pre-activation of lower limb musculature prior to ground contact [39]; neural drive from the H-reflex and the cortical center [32,40]; enhanced lower limb stiffness due to drop heights of less than $0.60 \mathrm{~m}[39,41]$; enhanced joint stiffness during the eccentric phase of the SSC which can enhance the storage and usage of elastic energy [42] which would enable the muscle to operate closer to its optimum length as well as shortening velocity [43]; increased activation and strength of the plantar flexor muscles and enhanced inter-muscular co-ordination [41].

An acute study examining attentional focus on DJ performance reported that the instruction "minimize ground contact time", induced stiffer landings due to augmented leg stiffness in comparison to other instructions ("maximize jump height" or "synchronously extend the lower extremity joints") [15].The result was the attenuation of DJ height but the enhancement of RSI, RFD, peak force and peak power. Our findings are contradictory for DJ height where we found a significant improvement which caused the significant enhancement of RSI in both groups of our study. Furthermore, enhancement of ECC-RFD, peak force and peak power were evident in the $\mathrm{CMJ}$ to a greater degree in the $\mathrm{COACH}$ group than the NONCOACH group which shows a transfer effect from the COACH group. Khuu et al. [15] reported attenuation of DJ height from the instruction "minimize ground contact time" which is in support of Young et al. [9] who reported DJ height was reduced when compared to the instruction, "maximize jump height". However, the instruction, "maximize jump height" leads to slower contact times changing the emphasis from a fast to a slow SSC. Previous research on BDJ programs targeting the fast SSC to enhance CMJ and sprint performance is due to the relationships between RSI, CMJ and sprint performance over 10 and $20 \mathrm{~m}$ $[28,44]$.

The BDJ training in the current study produced significant increases in 3RM back squat strength (absolute and relative) in both the NONCOACH $(\sim 6 \%)$ and COACH groups $(\sim 12.3 \%)$.This finding is in agreement with previous studies that employed DJs as a training tool to enhance the ability of muscle to develop force rapidly and to improve maximal rate of force development $[8,45]$. The significant improvement in 3RM back squat strength in both experimental groups in the current study is related to the augmentation of peak force and ECC-RFD in the CMJ. The coaching instructions employed in the $\mathrm{COACH}$ group led to a two-fold improvement in absolute and relative 3RM strength in comparison to the NONCOACH group. These coaching instructions in conjunction with an individualized drop height appears to have optimized the pre-activation of the lower limb musculature prior to ground contact and during eccentric loading at high velocity of the lower limbs during the eccentric phase of the BDJs. The pre-tensing or stiffening of the limbs prior to ground contact would enhance the pre-activation of the muscles before the eccentric phase which is a fundamental SSC condition [46]. When considering adaptations in fiber mechanics during eccentric muscle action, increased force generation may be due to mechanical detachment of cross-bridges and the activation of a second myosin head to act in increasing the number of active cross-bridges $[47,48]$. Furthermore, force potentiation is induced due to increased titin stiffness from the binding of calcium to titin [49]. The COACH group results confirm that the maximal activation of the lower limbs and the use of eccentric muscle actions by means of BDJs is important in causing force and power potentiation which augments $\mathrm{CMJ}$ performance in hurling players.

The significant improvement in maximum strength supported the augmentation of CMJ performance. ECC-RFD (absolute and relative) significantly improved pre- to post-BDJ training for the COACH group (Absolute ECC-RFD: 44.6\%; Relative ECC-RFD: $44.7 \%$ ). The following CMJ measures were augmented nonsignificantly pre- to post-training in the $\mathrm{COACH}$ group: height (7.5\%); absolute and relative peak force (13\%) and absolute and relative peak power (16\%). However, CMJ height $(9.5 \%)$ and peak force were augmented significantly from baseline to post-training 
(absolute peak force: $11.2 \%$; relative peak force: $10.1 \%$ ). A review reported that plyometric training can increase CMJ by $8.7 \%$ which compares favorably to the current study [50]. CMJ height augmentation is in agreement with previous studies that have employed training periods lasting 6 to 8-weeks using two sessions per week [5-9]. The augmentation of peak velocity and absolute and relative measures of peak force and peak power are similar to previous studies [6,7]. However, our results were non-significant for these measures in the COACH group pre- to post-training. ECCRFD was not compared as this is the first longitudinal plyometric study to the best of our knowledge to have measured RFD during a CMJ. The greater augmentation of force, power and ECC-RFD in the $\mathrm{CMJ}$ was because of the coaching instructions given to the COACH group. According to Stone et al. [51], learning to use an SSC, specifically a fast SSC in our study more proficiently, can lead to a marked enhancement in force production. To understand the mechanisms behind the effect of the eccentric phase of the fast SSC of the BDJ on CMJ measures where force peak, peak power and ECC-RFD were improved to a substantial degree, several are discussed. Firstly, increases in motor unit firing frequency can contribute to enhancements in eccentric power during a $\mathrm{CMJ}$ and supports the purported effects of motor unit discharge rates on eccentric strength [52]. Improvement in motor unit synchronization (intramuscular coordination) and inter muscular coordination (improved synergist co-activation and decreased antagonist co-activation) may also play a part in the expression of neuromuscular power [53].

The current study found no significant change in sprint performance $(5,10$ and $20 \mathrm{~m})$ pre to post the 6-week program for both groups. Our finding of a lack of improvement in the group response is in agreement with a previous study [54]. De Villareal et al. [8] stated that despite a significant improvement in $20 \mathrm{~m}$ performance, the improvement was rather small and suggested it was due to lack of specificity in their plyometric program. A possible reason for players not improving sprint performance but improving CMJ performance may be due to adaptations in their muscle architecture. Participants may have experienced an increase in pennation angle of the lateral gastrocnemiusover the course of the 6-week program which has been strongly related to jumping and not to sprint performance [55].

The major limitation of our study was the number of participants that withdrew at various stages due to injury or time constraints and not because of the experimental design. When considering the attentional focus strategy employed, future research may consider examining the use of specific external focus instructions in comparison to matched neutral or internal focus instructions for BDJs as the majority of previous research shows that external focus is the best approach. Furthermore, the frequency of instructions should be studied to determine how regularly athletes need to hear the instructions and probably should be related to the level of athlete targeted. Finally, examining the form in which coaching instructions are provided should be studied such as comparing verbal to the use of images and demonstrating the skill.

\section{Conclusion}

The current study used a novel approach to investigate how the effect of attentional focus during a 6-week BDJ program would transfer to strength, jumping and sprinting performance. Furthermore, this is the first BDJ longitudinal study using hurling players. The findings show that including neutral, internal and external focus coaching instructions leads to significant improvements in maximum lower limb strength, ECC-RFD, peak force and CMJ height. However, these coaching instructions did not lead to benefits for BDJ performance and so long as athletes know what is required during BDJ training the appropriate improvements in RSI, BDJ height and ground contact time should be observed. Therefore, coaches, athletes and sport science practitioners involved in hurling and other related field sports can expect to achieve improvements in strength, RFD and jumping from 60 bounce DJs performed twice a week for a short-term period when employing a combination of neutral, internal and external coaching instructions specific to BDJ training.

\section{Practical Applications}

Coaching athletes is critical to maintain a high level of training quality during a session. A combination of attentional focus coaching instructions during BDJ sessions is effective to enhance various forms of strength (maximum and explosive) and jumping performance. The coaching instructions employed appeared to have stimulated the athletes to maximize the eccentric phase of the DJs which led to greater improvements in peak force, peak power, ECC-RFD and CMJ height. It is important to highlight that athletes in this study completed two sessions per week of 60 DJs per session. Furthermore, the BDJ drop height was individualized using the RSI method to load the athletes appropriately during the eccentric phase of the jump as well as to prevent injury during these explosive movements. For coaches and athletes involved in hurling and other related field sports, the inclusion of external, internal and neutral coaching instructions during a BDJ program is valuable in providing a high quality training approach for strength and jumping improvements.

\section{References}

1. Lundin P (1985) Plyometrics: A review of plyometric training. Strength Cond J 7(3): 69-76.

2. Newton RU, Dugan E (2002) Application of Strength Diagnosis. Strength Cond J 24: 50-59.

3. Bobbert MF, Huijing PA, Van Ingen SG (1987) Drop jumping. I. The influence of jumping technique on the biomechanics of jumping. Med Sci Sports Exerc 19(4): 332-338.

4. Schmidtbleicher D (1992) Training for Power Events. In: Komi PV (Eds); Strength and power in sport. Blackwell, Boston, USA, pp. 381395.

5. Byrne PJ, Moran K, Rankin P (2010) A comparison of methods used to identify "optimal" drop height for early phase adaptations in depth jump training. J Strength Cond Res 24(5): 2050-2055.

6. Chelly MS, Ghenem MA, Abid K (2010) Effects of in-season shortterm plyometric training program on leg power, jump and sprint performance of soccer players. J Strength Cond Res 24(10): 2670-2676. 
7. Chelly MS, Hermassi S, Aouadi R (2014) Effects of 8-week in-season plyometric training on upper and lower limb performance of elite adolescent handball players. J Strength Cond Res 28(5): 1401-1410.

8. De Villareal ESS, Gonzalez-Badillo JJ, Izquierdo M (2008) Low and moderate plyometric training produces greater jumping and sprinting gains compared with high frequency. J Strength Cond Res 22(3): 715725 .

9. Young WB, Wilson GJ, Byrne C (1999) A comparison of drop jump training methods: Effects on leg extensor strength qualities and jumping performance. Int J Sports Med 20(5): 295-303.

10. Benz A, Winkelman N, Porter J, Nimphius S (2016) Coaching instructions and cues for enhancing sprint performance. Strength Cond J 38(1): 1-11.

11. Ives JC, Shelley GA (2003) Psychophysics in functional strength and power training: Review and implementation framework. J Strength Cond Res 17(1): 177-186.

12. Porter JM, Nolan RP, Ostrowski EJ (2010) Directing attention externally enhances agility performance: A qualitative and quantitative analysis of the efficacy of using verbal instructions to focus attention. Front Psychol 1: 1-7.

13. Wulf G, Prinz W, Hoß M (1998) Instructions for motor learning: Differential effects of internal versus external focus of attention. J Mot Behav 30(2): 169-179.

14. Young WB, Pryor JF, Wilson GJ (1995) Effect of instructions on characteristics of countermovement and drop jump performance. J Strength Cond Res 9(4): 232-236.

15. Khuu S, Musalem LL, Beach TAC (2015) Verbal instructions acutely affect drop vertical jump biomechanics-Implications for athletic performance and injury risk assessments. J Strength Cond Res 29(10): 2816-2826.

16. Porter JM, Ostrowski EJ, Nolan RP (2010) Standing long-jump performance is enhanced when using an external focus of attention. J Strength Cond Res 24(7): 1746-1750.

17. Wu WF, Porter JM, Brown LE (2012) Effect of attentional focus strategies on peak force and performance in the standing long jump. J Strength Cond Res 26(5): 1226-1231.

18. Wulf G, Dufek JS, Lozano L (2010) Increased jump height and reduced EMG activity with an external focus. Hum Mov Sci 29(3): 440-448.

19. Makaruk H, Porter JM, Czaplicki A (2012) The role of attentional focus in plyometric training. J Sports Med Phys Fitness 52(3): 319-327.

20. Flanagan EP, Comyns TM (2008) The use of contact time and the reactive strength index to optimize fast stretch-shortening cycle training. Strength Cond J 30(5): 32-38.

21. Turki O, Chaouachi A, Behm DG (2012) The effect of warm-ups incorporating different volumes of dynamic stretching on 10 and $20 \mathrm{~m}$ sprint performance in highly trained male athletes. J Strength Cond Res 26(1): 63-72.

22. Chatzinikolaou A, Fatouros IG, Gourgoulis V (2010) Time course of changes in performance and inflammatory responses after acute plyometric exercise. J Strength Cond Res 24(5): 1389-1398.

23. Lockie RG, Murphy AJ, Schultz AB (2012) The effects of different speed training protocols on sprint acceleration kinematics \& muscle strength and power in field sport athletes. J Strength Cond Res 26(6): 15391550.

24. Read MM, Cisar C (2001) The influence of varied rest interval lengths on depth jump performance. J Strength Cond Res 15(3): 279-283.

25. Laffaye G, Wagner PP, Tombleson TIL (2014) Countermovement jump height: gender and sport-specific differences in the force-time variables. J Strength Cond Res 28(4): 1096-1105.
26. Bosco C, Luhtanen P, Komi PV (1983) A simple method for measurement of mechanical power in jumping. Eur J Appl Physiol 50(2): 273-282.

27. Flanagan EP, Ebben WP, Jensen RL (2008) Reliability of the reactive strength index and time to stabilization during depth jumps. J Strength Cond Res 22(5): 1677-1682.

28. Cunningham DJ, West DJ, Owen NJ (2013) Strength and power predictors of sprinting performance in professional rugby players. J Sports Med Phys Fitness 53(2): 105-111.

29. Cohen J (1988) Statistical power analysis for the behavioral sciences $\left(2^{\text {nd }} e d n\right) ;$ L Erlbaum Associates Hillsdale, USA

30. Byrne PJ, Moody JA, Cooper SM (2017) The reliability of countermovement jump performance and the reactive strength index in identifying drop-jump drop height in hurling players. OAJ Exercise Sports Medicine 1(1): 1-10.

31. Ramirez-Campillo R, Alvarez C, García-Pinillos F (2018) Optimal Reactive Strength Index: Is It an Accurate Variable to Optimize Plyometric Training Effects on Measures of Physical Fitness in Young Soccer Players? J Strength Cond Res 32(4): 885-893.

32. Taube W, Leukel C, Lauber B (2012) The drop height determines neuromuscular adaptations and changes in jump performance in stretch-shortening cycle training. Scand J Med Sci Sports 22(5): 671683.

33. Poolton, JM, Maxwell, JP, Masters, RSW (2006) Benefits of an external focus of attention: Common coding or conscious processing? J Sports Sci 24(1): 89-99.

34. Wulf G, McNevin NH, Shea CH (2001) The automaticity of complex motor skill learning as a function of attentional focus. Quart J Exp Psych 54(4): 1143-1154.

35. Vance J, Wulf G, Töllner T (2004) EMG activity as a function of the performer's focus of attention. J Motor Behavior 36(4): 450-459.

36. Zachry T, Wulf G, Mercer J (2005) Increased movement accuracy and reduced EMG activity as the result of adopting an external focus of attention. Brain Res Bulletin 67(4): 304-309.

37. Ham DJ, Knez WL, Young WB (2007) A deterministic model of the vertical jump: Implications for training. J Strength Cond Res 21: $967-$ 972.

38. Newton RU, Rogers RA, Volek JS (2006) Four weeks of optimal load ballistic resistance training at the end of season attenuates declining jump performance of woman volleyball players. J Strength Cond Res 20(4): 955-961.

39. Kyrölänen H, Komi PV, Kim DH (1991) Effects of power training on neuromuscular performance and mechanical efficiency. Scand J Med Sci Sports 1(2): 78-87.

40.Zuur AT, Lundbye-Jensen J, Leukel C (2010) Contribution of afferent feedback and descending drive to human hopping. J Physiol 588(5): 799-807.

41. Kubo K, Morimoto M, Komuro T (2007) Effects of plyometric and weight training on muscle-tendon complex and jump performance. Med Sci Sports Exerc 39(10): 1801-1810.

42. Papadopoulos C, Theodosiou K, Bogdanis GC (2014) Multiarticular isokinetic high-load eccentric training induces large increases in eccentric and concentric strength and jumping performance.J Strength Cond Res 28(9): 2680-2688.

43. Cormie P, McGuigan MR, Newton RU (2010) Changes in the eccentric phase contribute to improved stretch-shorten cycle performance after training. Med Sci Sports Exerc 42(9): 1731-1744.

44. Shalfawi SAI, Sabbah A, Kailani G (2011) The relationship between running speed and measures of vertical jump in professional basketball players: A field-test approach. J Strength Cond Res 25(11): 3088-3092. 
45. Kramer JF, Morrow A, Leger A (1983) Changes in rowing ergometer, weight lifting, vertical jump and isokinetic performance in response to standard and standard plus plyometric training programs. Int J Sports Med 14(8): 449-454.

46. Komi PV, Gollhofer A (1997) Stretch reflex can have an important role in force enhancement during SSC exercise. J Appl Biomech 13(4): 451460 .

47. Linari M, Bottinelli R, Pellegrino MA (2004) The mechanism of the force response to stretch in human skinned muscle fibers with different myosin isoforms. J Physiol 554(2): 335-352.

48. Linari M, Lucii L, Reconditi M (2000) A combined mechanical and X-ray diffraction study of stretch potentiation in single frog muscle fibers. J Physiol 26(3): 589-596.

49. DuVall MM, Gifford JL, Amrein M (2013) Altered mechanical properties of titin immunoglobulin domain 27 in the presence of calcium. Eur Biophys J 42(4): 301-307
50. Markovic G (2007) Does plyometric training improve vertical jump height? A meta-analytical review. Br J Sports Med 41(6): 349-355.

51. Stone MH, Stone M, Sands WA (2007) Principles and practice of resistance training. Champaign, Human Kinetics, USA.

52. Aagaard P (2003) Training-induced changes in neural function. Exerc Sport Sci Rev 31(2): 61-67.

53. Cormie P, McGuigan MR, Newton RU (2011) Developing maximal neuromuscular power: Part 1. Biological basis of maximal power production. Sports Med 41(1): 17-38.

54. Markovic G, Jukic I, Milanovic D (2007) Effects of sprint and plyometric training on muscle function and athletic performance. J Strength Cond Res 21(2): 543-549.

55. Earp JE, Kraemer WJ, Newton RU (2010) Lower-body muscle structure and its role in jump performance during squat, countermovement, and depth drop jumps. J Strength Cond Res 24(3): 722-729.

\section{Your next submission with Juniper Publishers will reach you the below assets}

- Quality Editorial service

- Swift Peer Review

- Reprints availability

- E-prints Service

- Manuscript Podcast for convenient understanding

- Global attainment for your research

- Manuscript accessibility in different formats ( Pdf, E-pub, Full Text, Audio)

- Unceasing customer service

Track the below URL for one-step submission https://juniperpublishers.com/online-submission.php 